\section{Fatores associados à capacidade para o trabalho de trabalhadores do Setor Elétrico}

\author{
Factors associated with labor capacity in electric \\ industry workers
}

\author{
${ }^{1}$ Núcleo de Epidemiologia, \\ Hospital Samaritano de São \\ Paulo, São Paulo, Brasil. \\ 2 Faculdade de Saúde \\ Pública, Universidade de São \\ Paulo, São Paulo, Brasil. \\ Correspondência \\ M. C. Martinez \\ Núcleo de Epidemiologia, \\ Hospital Samaritano de \\ São Paulo. \\ Av. Onze de Junho 686, \\ apto. 61-B, São Paulo, SP \\ 04041-002, Brasil. \\ mcmarti@uol.com.br
}

\section{Abstract}

The Brazilian electric utility sector has recently undergone major industrial restructuring, with impacts on working conditions and work organization that could jeopardize the capacity for work. This study aimed to evaluate factors associated with labor capacity in a sample of 475 workers from an electric utility company in the State of São Paulo, Brazil. This cross-sectional study included descriptive and multiple linear regression analyses. The mean labor capacity index (LCI) was 41.8 points (on a scale from 7.0 to 49.0). Multiple regression showed that the factors that best explained LCI variability were work stress ( $p$ $<0.001)$ and physical health $(p<0.001$ in all the dimensions). In a separate analysis, excluding all the health dimensions, the variables associated with LCI were work stress ( $p<0.001)$, workplace ( $p=0.022)$, physical activity $(p=0.001)$, alcohol consumption ( $p=0.012)$, and body mass index $(p<0.001)$. The results highlighted aspects to be considered when developing measures to protect labor capacity, with an emphasis on stress prevention and health promotion.

Work Capacity Evaluation; Occupational Health; Working Conditions; Working Environment; Workload
Maria Carmen Martinez ${ }^{1}$

Maria do Rosário Dias de Oliveira Latorre 2

\section{Introdução}

O trabalho no Setor Elétrico destina-se à geração, transmissão e distribuição de energia elétrica 1,2 e é caracterizado pela presença de demandas físicas e mentais, co-existindo riscos à saúde e segurança dos trabalhadores que são de origem elétrica, mecânica, biológica, física, biomecânica e psicossocial 1,2,3. Como exemplo, um estudo junto a eletricitários do Estado do Rio de Janeiro, Brasil, cita, entre outras situações vivenciadas na execução do trabalho, o choque elétrico, as lesões de pés e mão por acidentes envolvendo manutenção de equipamentos e de rede elétrica, o esforço visual e mental na leitura e interpretação de plantas e croquis, as posturas inadequadas, os riscos de acidentes de trânsito, a exposição às condições climáticas, a ameaça de desemprego e a perda de benefícios anteriormente obtidos 1 .

A partir da segunda metade da década de 90, este setor passou por modificações caracterizadas pela privatização, redução de efetivos e reestruturação produtiva, com reflexos nas condições e na organização do trabalho, potencializando o efeito negativo dos riscos já existentes no trabalho. Neste contexto, diversas situações se tornaram recorrentes no desempenho das atividades laborais, tais como o aumento do volume de trabalho, intensificação de pressões de prazos e responsabilidades, exigência de maior qualificação profissional, instabilidade no emprego, 
maior competitividade e intensificação do uso da informação e de novas tecnologias 1,2,4.

A capacidade para o trabalho diz respeito à capacidade que o trabalhador tem para executar seu trabalho em função das exigências deste trabalho, de seu estado de saúde e de suas capacidades físicas e mentais 5 . Este conceito foi elaborado por pesquisadores do Finnish Institute of Occupational Health [Instituto Finlandês de Saúde Ocupacional], que estruturaram estudos sobre capacidade para o trabalho e envelhecimento funcional com base no modelo teórico estresse-desgaste de Rutenfranz ${ }^{6}$. Segundo este modelo, o desgaste do trabalhador é decorrente das cargas físicas e mentais do trabalho e é influenciado pela capacidade de enfrentamento do indivíduo, condicionando diferentes respostas ao estresse, podendo desencadear respostas fisiológicas, psicológicas e comportamentais, com efeitos negativos sobre a saúde e a capacidade para o trabalho 6,7. Esta resulta de um processo multicausal em que diversos elementos interagem, muitas vezes de maneira complexa 8,9,10. A partir deste referencial, foram desenvolvidos estudos de seguimento de populações de trabalhadores que contribuíram para identificar os determinantes de alterações na capacidade para o trabalho 11,12,13,14. Entre estes, podem ser citadas as características sócio-demográficas (sexo, idade, nível e competência educacional), os estilos de vida (prática de atividade física, obesidade, tabagismo e consumo de álcool), a saúde (percepção do estado de saúde, presença de sinais, sintomas e doenças, capacidade funcional física e mental) e o trabalho (condições e organização do trabalho) $11,15,16,17$.

Especificamente em relação aos eletricitários, apesar de executarem atividades que potencialmente podem levar a um comprometimento da capacidade para o trabalho, não foram identificados estudos abordando o tema entre esta categoria profissional. Assim, este estudo tem como objetivos analisar os fatores associadas à capacidade para o trabalho de eletricitários do Estado de São Paulo.

\section{Método}

Trata-se de estudo do tipo transversal realizado em uma empresa do Setor Elétrico da região de Campinas no Estado de São Paulo, Brasil, que passou por recente processo de reestruturação produtiva, acompanhado por mudanças nas condições e na organização do trabalho. $\mathrm{O}$ aperfeiçoamento dos processos de trabalho e a melhoria dos equipamentos contribuíram para a diminuição das cargas físicas, entretanto houve intensificação das cargas mentais em função de fatores como a ampliação das solicitações para análise de problemas e tomada de decisões, uso de novas tecnologias, intensificação de pressões de prazos e de responsabilidades e da instabilidade no emprego 18.

A população alvo foi composta pelos 582 trabalhadores do quadro de pessoal dos setores Transmissão de Energia, Estações Avançadas de Distribuição de Energia e Setor Administrativo (Recursos Humanos e Contabilidade). No setor de Transmissão de Energia ocorre a transmissão da energia das usinas hidrelétricas para subestações elevatórias da tensão e, em seguida, sua condução até estações rebaixadoras, a partir das quais o setor de Distribuição de Energia será responsável pela chegada da energia até o consumidor final por meio das redes elétricas 3,18 . O trabalho executado na Transmissão de Energia é caracterizado por exigências físicas e mentais, compreendendo atividades relativas à construção e manutenção das linhas de transmissão, incluindo estudos de planejamento, instalação e lançamento de estruturas, manutenção de equipamentos e desmatamento e limpeza de faixas de servidão 3,18. Na Distribuição de Energia destacam-se as atividades com exigências físicas e mentais, relacionadas à manutenção e limpeza dos equipamentos e redes de distribuição, limpeza das faixas de servidão, poda de árvores, medição de energia elétrica nos consumidores, operação dos centros de controle e de distribuição da energia, sendo estas atividades realizadas em sistemas energizados (linha viva) ou desenergizados 3,18. O setor Administrativo foi representado pelas áreas de Contabilidade e Recursos Humanos responsáveis, respectivamente, pelo fluxo de caixa, de contas a pagar e a receber, e pelo desenvolvimento de políticas, diretrizes e procedimentos de gestão do quadro de pessoal, predominando as exigências mentais 3,18 .

Os trabalhadores estão distribuídos em grande variedade de cargos que, para fins deste estudo, foram agrupados segundo as características das funções executadas 18. O cargo de "Liderança e Técnicos da Distribuição, Técnicos e Eletricistas da Transmissão" compreendeu os trabalhadores em atividades relativas predominantemente às tarefas especializadas e complexas em escritório ou campo, supervisão das equipes de campo e inspeção em subestações ou linhas de energia, podendo ainda executar ações de manutenção e manobras emergenciais ou programadas. Os trabalhadores com cargo "Eletricista de Distribuição" executam ações de inspeção e manutenção preventiva e corretiva de equipamentos, instalações e linhas de distribuição de energia. O cargo de "Eletricista de Linha Viva da Distribuição e da 
Transmissão" foi composto por trabalhadores que efetuam inspeção, manutenção e manobras em redes energizadas. O cargo "Administrativo" executa atividades de caráter técnico e gerencial realizadas em escritório.

Os critérios para exclusão do estudo foram estar em férias ou afastado do trabalho (por doença, acidente de trabalho ou em licença), sendo que $39(6,7 \%)$ dos trabalhadores se enquadraram nestes critérios. Das 543 pessoas elegíveis, houve 69 casos de perdas por estarem em cursos externos ou viagem a serviço, recusa na participação, respostas incompletas ou trabalhadores que não foram localizados. Restaram 474 pessoas, correspondendo a $82,3 \%$ dos trabalhadores que preencheram os critérios de inclusão. A análise das perdas não identificou diferenças estatisticamente significativas $(p>0,05)$ no que diz respeito ao sexo, setor de trabalho e tempo na empresa. Os dados para participantes e não participantes foram, respectivamente: $90,9 \%$ e $94,1 \%$ de homens ( $p=0,385), 66,5 \%$ e $66,2 \%$ do Setor de Distribuição de Energia ( $p=0,703$ ), e tempo médio de trabalho na empresa de 13,0 e $12,2$ anos ( $p=0,548)$. Houve uma diferença estatisticamente significativa $(p=0,030)$ em relação à idade: 37,5 e 39,7 anos.

A coleta de dados ocorreu em agosto de 2005, quando foram feitas reuniões com os empregados nas suas respectivas áreas de trabalho, visando a esclarecer quanto aos objetivos da pesquisa, aos aspectos éticos do estudo e à forma de coleta de dados. Nestas reuniões também foram entregues os questionários para serem auto-respondidos. Todas as reuniões foram conduzidas pela pesquisadora, que permaneceu no local para esclarecimento de dúvidas e recebimento dos questionários preenchidos.

Foi entregue um instrumento com cinco blocos de questionários. O primeiro destinava-se ao levantamento dos dados demográficos e funcionais. As variáveis demográficas foram sexo, idade, estado civil e escolaridade. As variáveis funcionais foram setor, cargo, unidade de trabalho (de acordo com a localização geográfica), turno, conteúdo do trabalho (físico, mental ou misto) e faixa salarial.

O segundo questionário foi a versão adaptada para uso no Brasil do Índice de Capacidade para o Trabalho (ICT) que permite avaliar a capacidade para o trabalho a partir da percepção do próprio trabalhador, fornecendo um escore que varia de 7 a 49 pontos, obtido a partir de dez questões (60 itens) sintetizadas em sete dimensões: (a) capacidade para o trabalho atual e comparada com a melhor de toda a vida, (b) capacidade para o trabalho em relação às exigências do trabalho, (c) número atual de do- enças auto-referidas e diagnosticadas por médico, (d) perda estimada para o trabalho devido a doenças, (e) falta ao trabalho por doenças, (f) prognóstico próprio sobre a capacidade para o trabalho e (g) recursos mentais 5 .

O terceiro questionário foi a Escala Estresse no Trabalho (EET), que fornece uma medida geral de estresse a partir da avaliação de 23 aspectos psicossociais do trabalho relativos à sobrecarga de trabalho, conflito entre papéis, ambigüidade de papéis, relacionamento interpessoal no trabalho e aspectos do desenvolvimento na carreira e autonomia/controle no trabalho 19. A EET gera um escore que varia de 1,0 a 5,0 pontos, e foi construída e validada para uso no Brasil 19.

O quarto questionário foi o Medical Outcomes Study 36 - Item Short Form Health Survey (SF-36) que é um questionário genérico de avaliação de saúde, traduzido e validado para o português e é composto por 36 itens englobados em oito escalas: capacidade funcional, aspecto físico, dor, estado geral de saúde, vitalidade, aspecto social, aspecto emocional e saúde mental 20 .

Para avaliação dos estilos de vida, foi utilizado o último bloco, composto por três questionários. Para avaliar a prática de atividade física foi utilizado o Questionário de Baecke, que investiga a atividade física habitual por meio de 16 questões relativas às atividades físicas ocupacionais, de lazer e de locomoção, e que foi validado para uso no Brasil 21. Para avaliar o estado nutricional foi usado o índice de massa corporal (IMC). O tabagismo foi avaliado por meio do Questionário de Tolerância de Fagerström para Nicotina, conforme recomendado pela Sociedade Brasileira de Cardiologia 22, pela Associação Médica Brasileira e pelo Conselho Regional de Medicina do Estado de São Paulo 23. Também foi utilizado o Alcohol Use Disorders Identification Test (AUDIT), que avalia dependência ao álcool, conforme preconizado pela Organização Mundial da Saúde (OMS) 24.

A consistência interna dos questionários foi avaliada por meio do coeficiente alpha de Cronbach. Para caracterização do perfil da população de estudo foi feita a análise descritiva por meio das médias, desvios-padrão, valores mínimos e máximos dos escores das variáveis contínuas e proporções para as variáveis qualitativas. Foi realizado o teste de Kolmogorov-Smirnov para verificar a aderência do escore do índice de capacidade para o trabalho à distribuição normal. Grande parte das variáveis não tinha distribuição normal e, por isso, na análise univariada foram utilizados testes não paramétricos.

Para análise da correlação entre a capacidade para o trabalho e as variáveis quantitativas foi usado o coeficiente de correlação de Spearman. 
Para comparação das médias do índice de capacidade para o trabalho segundo as categorias das variáveis qualitativas foram utilizados os testes Mann-Whitney e Kruskal-Wallis (com teste poshoc de Tukey). Foi realizada modelagem linear múltipla considerando o ICT como variável dependente. Foram selecionadas para a modelagem as variáveis que apresentaram $\mathrm{p}<0,20$ na análise univariada. O valor do "p" determinou a ordem de entrada no processo de modelagem que foi o stepwise forward. A variável independente permaneceu no modelo múltiplo se $\mathrm{p}<$ 0,05. Em todas as análises foi utilizado o nível de significância de $5 \%$.

Foram consideradas como variáveis de controle o sexo e o tempo de empresa. Verificou-se que os empregados que participaram da pesquisar eram mais jovens do que aqueles que não participaram. Com isso, seria ideal que a variável idade entrasse com controle. No entanto, ela apresentou forte correlação com o tempo de empresa $(\mathrm{r}=0,74 ; \mathrm{p}<0,001)$ e, por isso, optou-se por manter esta última no modelo. Esta decisão foi baseada no fato de o tempo de empresa retratar o desgaste decorrente do tempo de exposição às exigências do trabalho.

$\mathrm{O}$ projeto de pesquisa foi submetido à apreciação e aprovado pelo Comitê de Ética da Faculdade de Saúde Pública da Universidade de São Paulo, ele seguiu os princípios da Declaração de Helsinki, da World Medical Association [Associa- ção Médica Mundial]. A empresa onde ocorreu o estudo forneceu autorização formal para realização do mesmo. A participação dos trabalhadores foi voluntária e mediante assinatura do Termo de Consentimento Livre e Esclarecido. Os resultados individuais foram tratados com confidencialidade.

\section{Resultados}

Todas as escalas apresentaram boa consistência interna: ICT $\alpha=0,72$; Questionário de Baecke $\alpha=$ 0,66; Questionário de Fagerström $\alpha=0,69$; AUDIT $\alpha=0,79$; EET $\alpha=0,94$; e todas as escalas do SF-36 $\operatorname{com} \alpha>0,72$.

As características sócio-demográficas, dos estilos de vida, do trabalho e do estado de saúde da população de estudo estão apresentadas nas Tabelas 1 e 2 . A maior parte dos participantes foram homens $(90,9 \%)$, adultos jovens (média de idade $=36,8$ anos, $\mathrm{DP}=8,0$ ), casados(as) ou vivendo com companheira(o) $(75,2 \%)$, com Ensino Médio ou Superior completo (91,3\%). O tempo médio de trabalho na empresa foi de 12,8 $(\mathrm{DP}=7,5)$ anos. Os trabalhadores atuavam, principalmente, em turnos diurnos $(94,3 \%)$, predominantemente no Setor de Distribuição de Energia $(66,5 \%)$, ocupando cargos de Eletricista de Distribuição (50,5\%), em unidades de trabalho operacionais $(82,7 \%)$, desenvolvendo trabalhos

\begin{tabular}{|c|c|c|}
\hline Característica & $\mathbf{n}$ & $\%$ \\
\hline \multicolumn{3}{|l|}{ Sócio-demográficas } \\
\hline Homens & 432 & 90,9 \\
\hline Casado(a)/Vivendo com companheira(o) & 357 & 75,2 \\
\hline Ensino Médio completo & 307 & 64,6 \\
\hline Ensino Superior completo & 127 & 26,7 \\
\hline \multicolumn{3}{|l|}{ Relacionadas ao trabalho } \\
\hline Horário de trabalho diurno & 346 & 72,8 \\
\hline Setor de distribuição de energia & 316 & 66,5 \\
\hline Eletricistas de distribuição & 240 & 50,5 \\
\hline Conteúdo de trabalho misto (físico e mental) & 370 & 77,9 \\
\hline Unidades de trabalho operacionais & & 82,7 \\
\hline Renda mensal variando de 4,0 a 10,9 salários mínimos & 361 & 76,0 \\
\hline \multicolumn{3}{|l|}{ Estilos de vida } \\
\hline Tabagismo & 69 & 14,5 \\
\hline Consumo de álcool & 373 & 78,5 \\
\hline Consumo abusivo de álcool & 105 & 22,1 \\
\hline Sobrepeso ou obesidade & 284 & 59,8 \\
\hline
\end{tabular}


envolvendo tarefas com conteúdo físico e mental (77,9\%). A faixa de renda mensal predominantemente foi de 4,0 e 10,9 salários mínimos (76,0\%). O nível de estresse médio foi de 2,3 ( $\mathrm{DP}=0,7$ ) pontos em um escore de 1,0 a 7,0 pontos.

A grande maioria relatou algum tipo de prática regular de atividade física $(71,6 \%)$, com um escore médio 8,5 ( $\mathrm{DP}=1,3)$ pontos em uma escola de 0,0 a 15,0 pontos. O sobrepeso $(42,9 \%)$ e a obesidade $(16,8 \%)$ configuraram alterações do peso entre $59,7 \%$ dos trabalhadores. Poucos (14.5\%) eram tabagistas e $78,5 \%$ relataram consumo de bebidas alcoólicas, sendo que $22,1 \%$ apresentaram escore compatível com consumo sugestivo de dependência química.

As dimensões do estado que saúde que apresentaram maiores médias foram a capacidade funcional e o aspecto físico, com médias superiores a 88,0 em uma escala de 0,0 a 100,0 pontos.

A média do índice de capacidade para o trabalho foi de 41,8 (DP = 5,1) pontos em um escore de 7,0 a 49,0 , sendo que $25,1 \%$ dos trabalhadores apresentaram valores entre 46 e 49 pontos.

As doenças com maior prevalência, relatadas pelos trabalhadores, foram aquelas relacionadas a distúrbios músculo-esqueléticos: lesões nas costas $(26,7 \%)$, doença na parte superior das costas ou pescoço (20\%), lesões nos membros superiores $(19,2 \%)$. Os distúrbios emocionais leves também tiveram prevalência elevada $(22,1 \%)$.

Os resultados das análises univariadas dos fatores associados à capacidade para o trabalho estão apresentados nas Tabelas 3 e 4 . Fatores que apresentaram associação estatisticamente significativa com a capacidade para o trabalho foram: idade $(\mathrm{p}<0,001)$, unidade de trabalho $(\mathrm{p}=0,036)$, estresse no trabalho $(\mathrm{p}<0,001)$, índice de massa corporal $(\mathrm{p}<0,001)$, dependência química ao álcool $(\mathrm{p}=0,019)$, prática de atividade física $(\mathrm{p}<$ 0,001 ) e todas as dimensões do estado de saúde $(\mathrm{p}<0,001)$.

Foi feita uma primeira modelagem (Tabela 5, modelagem 1) que mostrou os seguintes fatores independentes associados à capacidade para 0 trabalho: estresse no trabalho, capacidade funcional, aspecto físico, dor e estado geral de saúde. Desta forma, quanto melhor a condição de saúde e quanto menor o nível de estresse no trabalho, maior o escore da capacidade para o trabalho. Este modelo apresentou coeficiente de determinação ajustado (rª) de 0,51, e a análise de resíduos mostrou homocedasticidade indicando ausência de vieses.

Como a saúde está conceitual e operacionalmente inclusa na estrutura do ICT, considerou-se que este modelo levou a uma perda de importância das demais variáveis em função da presença das dimensões da saúde. Com a finalidade de ve-
Tabela 2

Estatística descritiva das variáveis quantitativas. Eletricitários, região de Campinas, São Paulo, Brasil, 2005

\begin{tabular}{|c|c|c|}
\hline Característica & Média & Desvio-padrão \\
\hline \multicolumn{3}{|l|}{ Sócio-demográficas } \\
\hline Idade em anos & 36,8 & 8,0 \\
\hline \multicolumn{3}{|l|}{ Relacionadas ao trabalho } \\
\hline Tempo na empresa em anos & 12,8 & 7,5 \\
\hline Estresse no trabalho * & 2,3 & 0,7 \\
\hline \multicolumn{3}{|l|}{ Estilos de vida } \\
\hline Atividade física ** & 8,5 & 1,3 \\
\hline \multicolumn{3}{|l|}{ Estado de saúde *** } \\
\hline Capacidade funcional & 91,2 & 13,3 \\
\hline Aspecto físico & 88,3 & 24,4 \\
\hline Dor & 75,0 & 20,8 \\
\hline Estado geral de saúde & 80,7 & 16,1 \\
\hline Vitalidade & 72,8 & \\
\hline Aspecto social & 82,4 & 20,2 \\
\hline Aspecto emocional & 85,0 & 28,6 \\
\hline Saúde mental & 76,6 & 17,1 \\
\hline
\end{tabular}

rificar se outros fatores não passariam a ganhar significância no modelo final, foi realizada nova modelagem, agora sem as dimensões da saúde.

Nesta segunda modelagem (Tabela 5, modelagem 2) verificou-se que: quanto menor o estresse no trabalho, o IMC e a dependência ao álcool, mais elevado o escore do ICT; quanto maior a prática de atividade física, maior o escore do ICT; trabalhar na Unidade F representa diminuição no ICT quando comparada com as demais unidades de trabalho. Este modelo apresentou coeficiente de determinação ajustado ( $\left.\mathrm{r}^{2} \mathrm{a}\right)$ de 0,22 , e a análise de resíduos mostrou homocedasticidade indicando ausência de vieses.

\section{Discussão}

A taxa de participação no estudo foi de $82,3 \%$ e, portanto, acima dos $75 \%$ considerados como mínimos 25 . A análise da consistência interna demonstrou que os questionários utilizados tiveram confiabilidade satisfatória. Exceções foram os questionários de Baecke e de Fagerström, onde o alfa de Cronbach foi de 0,66 e 0,69, respectivamente. Considerando que a prática de atividade física e o tabagismo são relevantes para o estudo e que são construtos de mensuração complexa, optou-se por mantê-los na análise dos dados. 
Tabela 3

Análise das associações das variáveis qualitativas com a capacidade para o trabalho. Eletricitários, região de Campinas, São Paulo, Brasil, 2005.

\begin{tabular}{|c|c|c|c|}
\hline Variável/Categoria & $\mathrm{n}$ & Média (desvio-padrão) & Valor de $p$ \\
\hline \multicolumn{4}{|l|}{ Características sócio-demográficas } \\
\hline \multicolumn{4}{|l|}{ Sexo } \\
\hline Mulheres & 43 & $41,1(4,9)$ & 0,283 * \\
\hline Homens & 432 & $41,9(5,2)$ & \\
\hline \multicolumn{4}{|l|}{ Estado civil } \\
\hline Casado(a)/Com companheira(o) & 357 & $41,8(4,9)$ & 0,260 * \\
\hline Não casado(a) & 116 & $41,9(6,0)$ & \\
\hline \multicolumn{4}{|l|}{ Escolaridade } \\
\hline Sem Ensino Superior completo & 348 & $41,7(5,3)$ & 0,563 * \\
\hline Com Ensino Superior completo & 127 & $42,2(4,6)$ & \\
\hline \multicolumn{4}{|l|}{ Características relacionadas ao trabalho } \\
\hline \multicolumn{4}{|l|}{ Setor } \\
\hline Administrativo & 82 & $41,6(4,5)$ & $0,085 * \star$ \\
\hline Distribuição de energia & 316 & $41,6(5,4)$ & \\
\hline Transmissão de energia & 77 & $43,0(4,3)$ & \\
\hline \multicolumn{4}{|l|}{ Cargo } \\
\hline Administrativo & 90 & $41,5(4,5)$ & 0,257 ** \\
\hline Liderança e Técnicos da Distribuição, Técnicos e Eletricistas da Transmissão & 95 & $42,6(4,8)$ & \\
\hline Eletricista da Distribuição & 240 & $41,8(5,6)$ & \\
\hline Eletricista de Linha Viva da Distribuição e da Transmissão & 50 & $41,0(4,9)$ & \\
\hline \multicolumn{4}{|l|}{ Unidade de trabalho } \\
\hline A & 23 & $41,6(6,7)$ & 0,036 ** \\
\hline B & 41 & $41,2(6,1)$ & \\
\hline C & 50 & $42,7(3,7)$ & \\
\hline$D * \star \star$ & 44 & $43,5(3,7)$ & \\
\hline$E$ & 72 & $41,8(5,0)$ & \\
\hline $\mathrm{F}$ & 30 & $39,9(6,4)$ & \\
\hline G & 56 & $50,2(6,3)$ & \\
\hline$H^{\star \star \star}$ & 77 & $43,0(4,3)$ & \\
\hline I & 35 & $41,8(4,6)$ & \\
\hline$J$ & 47 & $41,4(4,5)$ & \\
\hline \multicolumn{4}{|l|}{ Turno } \\
\hline Diurno integral & 346 & $41,8(5,2)$ & 0,931 ** \\
\hline Matutino ou vespertino & 102 & $41,8(5,4)$ & \\
\hline Noturno & 27 & $42,3(3,6)$ & \\
\hline \multicolumn{4}{|l|}{ Conteúdo } \\
\hline Mental & 105 & $41,9(4,6)$ & 0,796 * \\
\hline Misto & 370 & $41,8(5,3)$ & \\
\hline \multicolumn{4}{|l|}{ Faixa salarial (salários mínimos) } \\
\hline$<4$ & 29 & $43,0(6,8)$ & $0,145 * \star$ \\
\hline $4,0-6,9$ & 218 & $41,3(5,5)$ & \\
\hline $7,0-10,9$ & 143 & $42,0(4,7)$ & \\
\hline $11-15,9$ & 36 & $41,9(5,1)$ & \\
\hline $16-20,9$ & 34 & $42,6(3,2)$ & \\
\hline $21-25,9$ & 15 & $43,7(3,9)$ & \\
\hline \multicolumn{4}{|l|}{ Estilos de vida } \\
\hline \multicolumn{4}{|l|}{ Dependência ao tabaco } \\
\hline Não fuma & 405 & $41,9(5,1)$ & $0,369 * \star$ \\
\hline Muito baixa, baixa ou média & 57 & $41,6(5,5)$ & \\
\hline Elevada ou muito elevada & 9 & $39,0(6,9)$ & \\
\hline
\end{tabular}

* Teste Mann-Whitney;

** Teste Kruskal-Wallis;

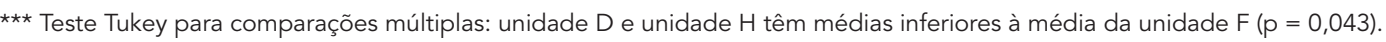


Coeficiente de correlação entre as variáveis e a capacidade para o trabalho. Eletricitários, região de Campinas, São Paulo, Brasil, 2005

\begin{tabular}{|c|c|c|c|}
\hline Variáveis & $\mathrm{n}$ & $\mathbf{r}$ & Valor de $p$ * \\
\hline \multicolumn{4}{|l|}{ Características demográficas } \\
\hline Idade & 475 & $-0,16$ & $<0,001$ \\
\hline \multicolumn{4}{|l|}{ Características relacionadas ao trabalho } \\
\hline Tempo na empresa & 475 & $-0,17$ & $<0,001$ \\
\hline Estresse no trabalho & 474 & $-0,37$ & $<0,001$ \\
\hline \multicolumn{4}{|l|}{ Estilos de vida } \\
\hline Índice de massa corporal $\left(\mathrm{kg} / \mathrm{m}^{2}\right)$ & 475 & $-0,17$ & $<0,001$ \\
\hline Consumo de álcool & 472 & $-0,11$ & 0,019 \\
\hline Prática de atividade física & 466 & 0,19 & $<0,001$ \\
\hline \multicolumn{4}{|l|}{ Estado de saúde } \\
\hline Capacidade funcional & 470 & 0,442 & $<0,001$ \\
\hline Aspecto físico & 474 & 0,317 & $<0,001$ \\
\hline Dor & 474 & 0,479 & $<0,001$ \\
\hline Estado geral de saúde & 473 & 0,491 & $<0,001$ \\
\hline Vitalidade & 475 & 0,394 & $<0,001$ \\
\hline Aspecto social & 475 & 0,334 & $<0,001$ \\
\hline Aspecto emocional & 474 & 0,231 & $<0,001$ \\
\hline Saúde mental & 475 & 0,395 & $<0,001$ \\
\hline
\end{tabular}

* Coeficiente de correlação de Spearman.

Tabela 5

Análise de regressão linear múltipla para identificação de fatores associados à capacidade para o trabalho. Eletricitários, região de Campinas, São Paulo, Brasil, 2005.

\begin{tabular}{|c|c|c|c|c|c|c|c|c|}
\hline \multirow[t]{2}{*}{ Variáveis } & \multicolumn{4}{|c|}{ Modelagem 1} & \multicolumn{4}{|c|}{ Modelagem 2} \\
\hline & b & IC95\% (b) & $\begin{array}{c}\text { Valor de p } \\
\text { (modelo) }\end{array}$ & $r^{2} a$ & b & IC95\% (b) & $\begin{array}{r}\text { Valor de p } \\
\text { (modelo) }\end{array}$ & $\mathrm{r} 2 \mathrm{a}$ \\
\hline Tempo na empresa (em anos) & $-0,021$ & {$[-0,066 ; 0,024]$} & 0,352 & 0,51 & $-0,047$ & {$[-0,103 ; 0,010]$} & 0,109 & 0,22 \\
\hline Estresse no trabalho (escore) & $-0,898$ & {$[-1,399 ;-0,396]$} & $<0,001$ & & $-2,411$ & {$[-2,998 ;-1,825]$} & $<0,001$ & \\
\hline Unidade de trabalho F & & & & & $-2,033$ & {$[-3,769 ;-0,298]$} & 0,022 & \\
\hline Prática de atividade física & & & & & 0,575 & {$[0,239 ; 0,911]$} & 0,001 & \\
\hline Consumo de álcool & & & & & $-0,126$ & {$[-0,224 ;-0,028]$} & 0,012 & \\
\hline Índice de massa corporal & & & & & $-0,204$ & {$[-0,316 ;-0,092]$} & $<0,001$ & \\
\hline Capacidade funcional (escore) * & 0,088 & {$[0,060 ; 0,117]$} & $<0,001$ & & & & & \\
\hline Aspecto físico (escore) * & 0,045 & {$[0,030 ; 0,061]$} & $<0,001$ & & & & & \\
\hline Dor (escore) * & 0,047 & {$[0,027 ; 0,066]$} & $<0,001$ & & & & & \\
\hline Estado geral de saúde (escore) * & 0,087 & {$[0,063 ; 0,111]$} & $<0,001$ & & & & & \\
\hline Sexo masculino & 0,973 & {$[-0,174 ; 2,120]$} & 0,096 & & 1,378 & {$[-0,170 ; 2,927]$} & 0,081 & \\
\hline
\end{tabular}

* Variáveis não incluídas na modelagem 2.

A população de estudo apresentou perfil sócio-demográfico, dos estilos de vida e do estado de saúde com melhores resultados do que a população geral de São Paulo, sendo que uma discussão detalhada sobre estes resultados está apresentada em outra publicação ${ }^{18}$. Cabe ressaltar que a população de estudo teve melhor padrão do que a população geral no que diz 
respeito à remuneração e ao nível de escolaridade, e que este padrão diferenciado também foi identificado em um estudo junto aos eletricitários do Rio de Janeiro ${ }^{26}$. A comparação com outros estudos 27,28,29,30 também mostrou perfil mais favorável da população de estudo quanto ao tabagismo e à prática de atividades físicas.

As prevalências de sobrepeso e obesidade foram elevadas, mas é difícil estabelecer comparações com outros grupos populacionais dada a grande divergência de resultados, com variações de $13,7 \%$ a $29,4 \%$ somente na obesidade $27,28,29,30$

O perfil de algum grau de uso $(78,5 \%)$ e abuso $(22,1 \%)$ de álcool entre os trabalhadores da população de estudo foi desfavorável em relação aos resultados de um inquérito populacional onde 71,5\% da população da Região Sudeste do Brasil consome bebidas alcoólicas, com 9,2\% com características compatíveis com dependência química ao álcool 31 .

Os escores do SF-36 mostraram boa condição de saúde, sendo que as dimensões vitalidade, percepção de dor e saúde mental foram aquelas com pior desempenho, apesar dos resultados sempre superiores a $70 \%$ do valor máximo dos escores.

O estresse decorrente de fatores do ambiente psicossocial do trabalho esteve em nível intermediário, e este resultado foi similar ao obtido em um estudo usando a mesma metodologia para avaliar 237 trabalhadores de uma instituição bancária 19

Os eletricitários apresentaram valores elevados em todas as dimensões que compõem o ICT, com um resultado final de 41,8 pontos em um escore que varia de 13,0 a 49,0. Esta média foi um pouco inferior à encontrada em estudos junto a outros grupos de trabalhadores da região de Campinas: 42,0 (DP $=4,5)$ pontos em trabalhadores de enfermagem 32 e 42,7 $(\mathrm{DP}=4,2)$ em trabalhadores de tecnologia de informação 33. Entretanto, comparações entre diferentes grupos laborais requerem uma padronização da população e devem analisadas com cautela, pois diferenças na composição demográfica podem gerar vieses em função de especificidades determinadas por questões de gênero e etárias.

O elevado nível de saúde e de capacidade para o trabalho observado nesta população de estudo pode sofrer influência do efeito do trabalhador sadio. Esse efeito é apontado em outros estudos sobre capacidade para o trabalho como responsável por possíveis distorções nos perfis e associações identificados 10,34. Estudos junto a eletricitários também apontam o efeito do trabalhador sadio, das condições sócio-econômi- cas e de inserção laboral destes trabalhadores como influenciando resultados 22,35,36.

Os distúrbios músculo-esqueléticos e os emocionais leves foram os agravos identificados por meio do ICT que apareceram com maiores prevalências. As lesões músculo-esqueléticas podem estar relacionadas tanto às exigências físicas do trabalho de campo como ao uso do computador pelo pessoal administrativo, e os distúrbios emocionais leves podem estar relacionados à reestruturação produtiva que ocorreu na empresa. Esse resultado é compatível com as mudanças que vêm ocorrendo no perfil epidemiológico dos trabalhadores no Brasil e no mundo, como conseqüência da introdução das novas tecnologias, das mudanças nos processos de trabalho e da flexibilização nas relações trabalhistas. Nas últimas décadas vem aumentando a relevância dos agravos com etiologia multicausal, destacando-se as doenças músculo-esqueléticas relacionadas ao trabalho, o comprometimento da saúde mental e os sintomas inespecíficos e/ou co-existentes relacionados ao estresse no trabalho 12,37,38,39.

A capacidade para o trabalho, considerada como resultante de um processo dinâmico entre recursos do indivíduo em relação ao seu trabalho, sofre modificações em função de vários fatores, entre eles a condição de saúde, as características sócio-demográficas, os estilos de vida, o envelhecimento e os fatores relacionados ao trabalho 11,15,16,17. Nesta população de estudo, as características demográficas não estiveram associadas à capacidade para o trabalho. Cabe ressaltar a questão da idade, dado que o envelhecimento cronológico é considerado como um dos determinantes do envelhecimento funcional 9,15,17,39. Neste estudo a idade esteve inversamente associada à capacidade para o trabalho, mas perdeu seu significado estatístico quando da análise múltipla. Isso se deu porque a idade teve forte correlação com o tempo de empresa $(\mathrm{r}=0,74, \mathrm{p}<0,001)$, que foi priorizado na análise por ser uma variável que representaria exposição às exigências do trabalho ao longo da vida do trabalhador.

A análise das variáveis unidade de trabalho, setor, cargo, turno, conteúdo do trabalho e rendimentos investigou se características como exigências, condições e organização do trabalho relativos a cada uma das variáveis estariam associadas à capacidade para o trabalho. Somente a unidade de trabalho, o tempo de empresa e o estresse no trabalho estiveram associadas à capacidade para o trabalho e, na análise múltipla, o tempo de empresa perdeu a significância estatística.

Alguns aspectos podem ser apontados como possíveis explicações para estes resultados. 
O efeito do trabalhador sadio, determinado pela exclusão daqueles trabalhadores com capacidade para o trabalho já comprometida em função de cargas originadas pelo conteúdo, turno ou outras características do contexto laboral, faria com que estas características não apresentassem relevância entre os remanescentes na ocasião do estudo. Outra hipótese explicativa é que, apesar das mudanças organizacionais ocorridas, os trabalhadores remanescentes tiveram preservados seus benefícios, direitos trabalhistas e acesso a programas de saúde e segurança. Esta situação, aliada às características sócio-demográficas desta população configurariam, no coletivo dos trabalhadores, uma homogeneidade que prevaleceria sobre as características específicas relativas às funções desempenhadas.

Quanto à unidade de trabalho (localização geográfica), os trabalhadores da unidade F apresentaram pior condição da capacidade para o trabalho quando comparados aos trabalhadores das unidades D e H. Entretanto, as análises realizadas não permitiram identificar os motivos desta diferença.

O nível de estresse decorrente dos fatores psicossociais do ambiente de trabalho apareceu inversamente associado ao nível da capacidade para o trabalho, independente das questões de saúde e dos estilos de vida. No intuito de verificar a existência de diferenças no nível de estresse decorrentes de características do trabalho, realizou-se uma comparação entre médias que evidenciou a não existência de associação entre estresse e setor $(p=0,601)$, cargo $(p=0,752)$, unidade de trabalho $(\mathrm{p}=0,378)$, turno de trabalho $(p=0,302)$, conteúdo do trabalho $(p=0,952)$ e faixa salarial $(p=0,571)$. Esta última análise mostrou que o estresse se apresentou de forma homogênea entre os trabalhadores, independente destas características funcionais.

Nas últimas décadas, a organização, os métodos, as ferramentas e as cargas de trabalho vêm mudando mais rapidamente do que a capacidade de adaptação humana, representando uma das principais causas de mudanças negativas na capacidade para o trabalho ${ }^{6}$. $O$ estresse decorrente do esforço físico está consistentemente demonstrado como fator de risco para a capacidade para o trabalho 12,13,15. Estudos também têm evidenciado que o estresse decorrente de fatores da organização e do ambiente psicossocial do trabalho exerce efeitos importantes sobre a capacidade para o trabalho em diferentes tipos de atividade 16,39,40,41.

Embora o desenho desta pesquisa não permita comprovar esta afirmação, pode-se especular que este resultado esteja relacionado ao contexto da empresa onde ocorreu o estudo, que pode ter sofrido os impactos do processo de reestruturação produtiva e tecnológica que ocorreu no Setor Elétrico no país. Nesse processo foram recorrentes as situações de aumento das pressões de prazos, volume de trabalho e responsabilidades, insegurança quanto ao futuro na empresa, exigência de maior diversidade de soluções, adaptação a novas tecnologias e demanda de maior qualificação técnico-profissional, com profundas modificações nas condições e na organização do trabalho, nas relações de trabalho e na inserção do trabalhador no mercado de trabalho, sendo apontado como origem de conseqüências negativas para a saúde e bem-estar dos trabalhadores das empresas do setor 1,2,18.

Apesar de o nível de estresse identificado poder ser considerado relativamente moderado, sua associação com a capacidade para o trabalho foi demonstrada e, além disso, ele deve ser considerado por exercer detrimento sobre a saúde dos trabalhadores. Além dos efeitos imediatos, o estresse também pode ter efeitos de médio e longo prazo. Exposição contínua a condições adversas pode desencadear prejuízos em termos de morbidade, comprometimento do bem-estar, redução da produtividade, aumento do absenteísmo e da insatisfação no trabalho 17,37,41,42.

Entre os trabalhadores da população de estudo, a elevação do padrão de prática de atividade física esteve significativamente associada à elevação do índice de capacidade para o trabalho. Os efeitos benéficos da atividade física sobre a capacidade para o trabalho de diferentes grupos de trabalhadores podem se dar por meio do aumento do consumo energético diário, redução da gordura corporal, manutenção da capacidade aeróbia, aumento da resistência e da força muscular e melhor percepção do estado de saúde 14,34. Esta questão merece atenção especial quando se trata de trabalhadores onde existe a necessidade de um bom preparo físico como recurso para preservar a qualificação para atender às exigências físicas nas tarefas, como é o caso dos eletricitários.

O consumo de álcool esteve inversamente relacionado à capacidade para o trabalho. O papel do álcool estaria relacionado à influência que o mesmo exerce sobre a condição física e mental dos trabalhadores, decorrente tanto do uso crônico como das intoxicações agudas 23. Padrões sociais levam o trabalhador a ocultar seu padrão de consumo alcoólico no trabalho quando este se torna abusivo 23,43 . Assim, a já elevada prevalência de trabalhadores com consumo alcoólico abusivo que foi identificada neste estudo pode estar subestimada.

O IMC esteve inversamente correlacionado ao ICT. O excesso de peso exerce impacto negati- 
vo sobre a capacidade para o trabalho por afetar a capacidade cardiorrespiratória e músculo-esquelética e por favorecer o aumento da morbidade decorrente de doenças crônicas como as cardiopatias, diabetes e depressão ${ }^{9,34}$. No caso dos eletricitários, o excesso de peso associado à diminuição da capacidade para o trabalho pode ser um problema especialmente relevante para os trabalhadores que executam atividades com exigências físicas, onde há necessidade de boa condição da capacidade funcional.

Apesar de todas as dimensões do SF-36 estarem positivamente correlacionadas ao ICT, na primeira modelagem múltipla somente as dimensões representativas da saúde física permaneceram associadas à capacidade para o trabalho. Este resultado é entendido sob dois aspectos. Primeiro quanto à saúde mental: as quatro dimensões representativas da saúde mental perderam o significado estatístico quando foi introduzida a variável estresse no trabalho, mostrando que a associação entre saúde mental e capacidade para o trabalho é dependente do estresse, reforçando o papel deste.

O segundo aspecto é relativo à saúde física. O papel da saúde física sobre a capacidade para o trabalho está consistentemente demonstrado, sendo a capacidade cardiorrespiratória, o funcionamento músculo-esquelético e a presença de sintomas e de doenças os aspectos que exercem maior impacto 13,34,39,40. Neste estudo, os resultados mostraram que a possibilidade de desempenhar atividades físicas sem limitações devidas à dor ou à alteração do funcionamento do organismo, a ausência de doenças e uma boa percepção do estado geral de saúde foram atributos importantes relacionados à capacidade para o trabalho. Esses resultados expressam a relevância de uma boa qualidade do estado de saúde físico para o desempenho das atividades laborais do grupo de eletricitários estudado. Estas atividades são predominantemente caracterizadas como tendo conteúdo misto (físico e mental), com algumas das tarefas podendo apresentar exigências físicas bastante intensas e/ou freqüentes 18. Desta forma, as características dos estilos de vida já citadas ganharam relevância no 2o modelo estatístico realizado, possivelmente por seu papel reconhecidamente determinante do estado de saúde.

Concluindo, os resultados permitiram identificar aspectos que devem ser considerados na concepção e desenvolvimento de medidas visando a preservação da capacidade para o trabalho, com ênfase no controle do estresse relacionado aos fatores do ambiente psicossocial do trabalho e na promoção, proteção e recuperação da saúde por meio da prevenção e tratamento da dependência ao álcool, incentivo à prática de atividade física e controle de peso. O estresse no trabalho foi um fator especialmente relevante, pois manteve sua associação com a capacidade para o trabalho independente das questões de saúde e de estilos de vida. 


\section{Resumo}

O Setor Elétrico passou por recente processo de reestruturação produtiva com reflexos nas condições e organização do trabalho, podendo comprometer a capacidade para o trabalho. Este estudo objetivou avaliar fatores associados à capacidade para o trabalho junto a 475 trabalhadores de uma empresa do Setor Elétrico no Estado de São Paulo, Brasil. Neste estudo transversal foi realizada análise descritiva e análise de regressão linear múltipla. A média do Índice de Capacidade para o Trabalho (ICT) foi de 41,8 pontos (escala de 7,0 a 49,0 pontos). A análise múltipla mostrou que os fatores que melhor explicaram a variabilidade do ICT foram estresse no trabalho $(p<0,001)$ e saúde física $(p<0,001$ em todas as dimensões). Em outra análise, excluídas as dimensões da saúde, as variáveis associadas ao ICT foram estresse no trabalho $(p<0,001)$, local de trabalho $(p=0,022)$, prática de atividade física ( $p=$ $0,001)$, consumo de álcool $(p=0,012)$ e indice de massa corporal $(p<0,001)$. Os resultados identificaram aspectos a serem considerados no desenvolvimento de medidas visando a preservação da capacidade para o trabalho, com ênfase no controle do estresse no trabalho e na promoção da saúde.

Avaliação da Capacidade de Trabalho; Saúde do Trabalhador; Condições de Trabalho; Ambiente de Trabalho; Carga de Trabalho

\section{Colaboradores}

M. C. Martinez foi responsável pelo planejamento da pesquisa, revisão bibliográfica, trabalho de campo, digitação e análise dos dados e redação do manuscrito. M. R. D. O. Latorre participou na orientação geral e no planejamento da pesquisa, bem como na análise dos dados, e contribuiu na estruturação e revisão do manuscrito.

\section{Referências}

1. Nogueira VA. Reestruturação do Setor Elétrico: um estudo qualitativo das condições de trabalho e saúde dos eletricitários frente à privatização da CERJ [Dissertação de Mestrado]. Rio de Janeiro: Escola Nacional de Saúde Pública, Fundação Oswaldo Cruz; 1999.

2. Guimarães LBM, Fischer D, Fae CS, Salis HB, Santos JAS. Apreciação macroergonômica em uma concessionária de energia elétrica. In: Anais do VII Congresso Latino-Americano, XII Congresso Brasileiro de Ergonomia e I Seminário Brasileiro de Acessibilidade Integral [CD-ROM]. Recife: Associação Brasileira de Ergonomia; 2002.

3. Departamento de Segurança e Saúde no Trabalho, Ministério do Trabalho e Emprego. Manual setor elétrico e telefonia. Brasília: Ministério do Trabalho e Emprego; 2002.

4. Departamento Intersindical de Estatística e Estudos Sócio-Econômicos. Emprego e desemprego no Setor Elétrico. São Paulo: Departamento Intersindical de Estatística e Estudos Sócio-Econômicos; 1999.

5. Tuomi K, Ilmarinen J, Jahkola A, Katajarinne L, Tulkki A. Índice de capacidade para o trabalho. São Carlos: Editora da Universidade Federal de São Carlos; 2005.
6. Ilmarinen J, Tuomi K, Eskelinen L, Nygård C-H, Huuhtanen P, Klockars M. Background and objectives of the Finnish research project on aging workers in municipal occupations. Scand J Work Environ Health 1991; 17 Suppl 1:7-11.

7. Colquhoun WP, Rutenfranz J. Studies of shiftwork. London: Taylor \& Francis; 1980.

8. Ilmarinen J. Aging and work. Occup Environ Med 2001; 58:546-51.

9. Ilmarinen J. Towards a longer worklife! Ageing and the quality of worklife in the European Union. Helsinki: Finnish Institute of Occupational Health; 2006.

10. Costa G. Some considerations about aging, shift work and work ability. In: Costa G, Goedhart WJA, Ilmarinen J, editors. Proceedings of the 2nd International Symposium in Work Ability: assessment and promotion of work ability, health and well-being of ageing workers. Amsterdam: Elsevier; 2005. p. 67-72. (International Congress Series, 1280).

11. Ilmarinen J, Tuomi K, Klockars M. Changes in the work ability of active employees over an 11-year period. Scand J Work Environ Health 1997; 17 Suppl 1:49-57. 
12. Tuomi K, Vanhala S, Nykyri E, Janhonen M. Organizational practices, work demands and the well-being of employees: a follow-up study in the metal industry and retail trade. Occup Med 2004; 54:115-21.

13. Savinainen M, Nygård C-H, Ilmarinen J. Workload and physical capacity among ageing municipal employees: a 16-year follow-up study. Int J Ind Ergon 2004; 34:519-33.

14. Smolander J, Blair SN, Kohl HW. Work ability, physical activity, and cardiorespiratory fitness: 2-year results from project active. J Occup Environ Med 2000; 42:906-10.

15. Tuomi K, Eskelinen L, Toikkanen J, Jarvinen E, Ilmarinen J, Klockars M. Work load and individual factors affecting work ability among aging municipal employees. Scand J Work Environ Health 1991; 17 Suppl 1:28-34.

16. Tuomi K, Ilmarinen J, Martikainen R. Aging, work, life-style and work ability among Finnish municipal workers in 1981-1992. Scand J Work Environ Health 1997; 23 Suppl 1:58-65.

17. Tuomi K, Ilmarinen J, Seitsamo J, Huuhtanen P, Martikainen R, Nygård C-H, et al. Summary of the Finnish research project (1981-1992) to promote the health and work ability of aging workers. Scand J Work Environ Health 1997; 23 Suppl 1:66-71.

18. Martinez MC, Latorre MRDO. Saúde e capacidade para o trabalho de eletricitários do Estado de São Paulo. Ciênc Saúde Coletiva 2008; 13:1061-73.

19. Paschoal T, Tamayo A. Validação da Escala de Estresse no Trabalho. Estud Psicol (Natal) 2004; 9:45-52.

20. Ciconelli RM, Ferraz MB, Santos W, Meinão I, Quaresma MR. Tradução para a língua portuguesa e validação do questionário genérico de avaliação de/da qualidade de vida SF-36 (Brasil SF-36). Rev Bras Reumatol 1999; 39:143-50.

21. Florindo AA, Latorre MRDO. Validation and reliability of the Baecke questionnaire for the evaluation of habitual physical activity in adult men. Rev Bras Med Esporte 2003; 9:129-35.

22. Sociedade Brasileira de Cardiologia. III Diretrizes brasileiras sobre dislipidemias e diretriz de prevenção da aterosclerose do Departamento de Aterosclerose da Sociedade Brasileira de Cardiologia. Arq Bras Cardiol 2001; 77 Suppl 3:1-48.

23. Laranjeira R, coordenador. Usuários de substâncias psicoativas: abordagem, diagnóstico e tratamento. São Paulo: Conselho Regional de Medicina do Estado de São Paulo/Associação Médica Brasileira; 2002.

24. World Health Organization. Management of substance dependence: screening and brief intervention. Geneva: World Health Organization; 2003.

25. Fowler Jr. DJ. Survey research methods. Thousand Oaks: Sage Publications; 1990.

26. Mattos IE, Sauaia N, Menezes PR. Padrão de mortalidade por câncer em trabalhadores eletricitários. Cad Saúde Pública 2002; 18:221-33.

27. Simão M, Nogueira MS, Hayashida M, Cesarino EJ. Doenças cardiovasculares: perfil de trabalhadores do sexo masculino de uma destilaria do interior paulista. Rev Eletrônica Enferm 2002; 4:27-35.

28. Marcopito LF, Rodrigues SSF, Pacheco MA, Shirassu M, Goldfeder AJ, Moraes MA. Prevalência de alguns fatores de risco para doenças crônicas na cidade de São Paulo. Rev Saúde Pública 2005; 39:738-45.
29. Barberino J, Carvalho F, Silvany-Neto A, Cotrim H, Góes R, Rosa H, et al. Alterações hepáticas em trabalhadores de uma refinaria de petróleo e em uma população de referência no Estado da Bahia, Brasil. Rev Panam Salud Pública 2005; 17:30-7.

30. Coordenação de Prevenção e Vigilância, Instituto Nacional de Câncer. Inquérito domiciliar sobre comportamentos de risco e morbidade referida de doenças e agravos não transmissíveis: Brasil, 15 capitais e Distrito Federal, 2002-2003. Rio de Janeiro: Instituto Nacional de Câncer; 2004.

31. Carlini EA, Galduróz JCF, Noto AR, Nappo AS. I levantamento domiciliar sobre o uso de drogas psicotrópicas no Brasil: estudo envolvendo as 107 maiores cidades do país - 2001. São Paulo: Centro Brasileiro de Informações Sobre Drogas Psicotrópicas/Universidade Federal de São Paulo; 2002.

32. Duran ECM, Cocco MIM. Capacidade para o trabalho entre trabalhadores de enfermagem do pronto socorro de um hospital universitário. Rev Latinoam Enferm 2004; 12:43-9.

33. Monteiro MI, Fernandes ACP. Capacidade para o trabalho de trabalhadores de empresa de tecnologia da informação. Rev Bras Enferm 2006; 59:603-8.

34. Pohjonen T. Age-related physical fitness and the predictive values of fitness testes for work ability in home care work. J Occup Environ Med 2001; 43:723-30.

35. Kelsh MA, Sahl JD. Mortality among a cohort of electric utility workers, 1960-1991. Am J Ind Med 1997; 31:534-44.

36. Nichols L, Sorahan T. Mortality of UK electricity generation and transmission workers, 1973-2002. Occup Med 2005; 55:541-8.

37. Karasek R, Theorell T. Healthy work: stress, productivity, and the reconstruction of working life. New York: Basic Books; 1990.

38. Wünsch Filho V. Perfil epidemiológico dos trabalhadores. Rev Bras Med Trab 2004; 2:103-17.

39. Ilmarinen JI, Tuomi K, Seitsamo J. New dimensions of work ability. In: Proceedings of the $2^{\text {nd }}$ International Symposium in Work Ability: assessment and promotion of work ability, health and well-being of ageing workers. Amsterdam: Elsevier; 2005. p. 3-7. (International Congress Series, 1280).

40. Sjögren-Rönkä T, Ojanen MT, Leskinen EK, Mustalampi ST, Mälkiä EA. Physical and psychosocial prerequisites of functioning in relation to work ability and general subjective well-being among office workers. Scand J Work Environ Health 2002; 28:184-90.

41. Goedhard RG, Goedhard WJA. Work ability and perceived work stress. In: Proceedings of the 2nd International Symposium in Work Ability: assessment and promotion of work ability, health and well-being of ageing workers. Amsterdam: Elsevier; 2005. p. 79-83. (International Congress Series, 1280).

42. Stansfeld S, Candy B. Psychosocial work environment and mental health - a meta-analytic review. Scand J Work Environ Health 2006; 32:443-62.

43. Neves DP. Alcoolismo: acusação ou diagnóstico? Cad Saúde Pública 2004; 20:7-36.

Recebido em 27/Jun/2008

Versão final reapresentada em 22/Set/2008 Aprovado em 01/Dez/2008 